PROCEEDINGS OF THE

AMERICAN MATHEMATICAL SOCIETY

Volume 137, Number 1, January 2009, Pages 135-143

S 0002-9939(08)09668-8

Article electronically published on August 26, 2008

\title{
A NOTE ON THE CONE RESTRICTION CONJECTURE IN THE CYLINDRICALLY SYMMETRIC CASE
}

\author{
SHUANGLIN SHAO \\ (Communicated by Hart F. Smith)
}

\begin{abstract}
In this paper, we present two arguments showing that the classical linear adjoint cone restriction conjecture holds for the class of functions supported on the cone and invariant under spatial rotation in all dimensions. The first is based on a dyadic restriction estimate, while the second follows from a strengthening version of the Hausdorff-Young inequality and the Hölder inequality in Lorentz spaces.
\end{abstract}

\section{INTRODUCTION}

Let $n \geq 2$ be a fixed integer and $S$ be a smooth compact non-empty subset of the cone $\left\{(\tau, \xi) \in \mathbf{R} \times \mathbf{R}^{n}: \tau=|\xi|\right\}$, where we interpret $\mathbf{R} \times \mathbf{R}^{n}$ as the time-space frequency space. If $0<p, q \leq \infty$, the classical linear adjoint restriction estimat 11 for the cone is the following "a priori" estimate:

$$
\left\|(f d \sigma)^{\vee}\right\|_{L_{t, x}^{q}\left(\mathbf{R} \times \mathbf{R}^{n}\right)} \leq C_{p, q, n, S}\|f\|_{L^{p}(S, d \sigma)}
$$

for all Schwartz functions $f$ on $S$, where

$$
(f d \sigma)^{\vee}(t, x)=\int_{S} f(\tau, \xi) e^{i(x \cdot \xi+t \tau)} d \sigma(\xi)=\int_{\mathbf{R}^{n}} f(|\xi|, \xi) e^{i(x \cdot \xi+t|\xi|)} \frac{d \xi}{|\xi|}
$$

denotes the inverse time-space Fourier transform of the measure $f d \sigma$, and $d \sigma$ is the pull-back of the measure $\frac{d \xi}{|\xi|}$ under the projection map $(\tau, \xi) \mapsto \xi$. By duality, the estimate (1.1) is equivalent to

$$
\|\hat{f}\|_{L^{p^{\prime}(S, d \sigma)}} \leq C_{p, q, n, S}\|f\|_{L^{q^{\prime}}\left(\mathbf{R} \times \mathbf{R}^{n}\right)}
$$

for all Schwartz functions $f$, which roughly says that the Fourier transform of an $L^{q^{\prime}}\left(\mathbf{R} \times \mathbf{R}^{n}\right)$ function can be "meaningfully" restricted to the cone $S$. This leads to the restriction problem, one of the central problems in harmonic analysis, which concerns the optimal range of exponents $p$ and $q$ for which the estimate (1.1) should hold. It was originally proposed by Stein for the sphere [5] and then extended to smooth submanifolds of $\mathbf{R} \times \mathbf{R}^{n}$ with appropriate curvature [6. Chapter 8, pages $352-355,364-367]$ such as the paraboloid and the cone. The restriction problem is intricately related to other outstanding problems in analysis such as the BochnerRiesz conjecture, the Sogge local smoothing conjecture, the Kakeya set conjecture and the Kakeya maximal function conjecture; see, e.g., 9], 10].

Received by the editors October 9, 2007.

2000 Mathematics Subject Classification. Primary 42B10, 42B25; Secondary 35L05.

${ }^{1}$ In the notation of [9], the estimate (1.1) is denoted by $R_{S}^{*}(p \rightarrow q)$.

(C)2008 American Mathematical Society 135

Reverts to public domain 28 years from publication 
By testing (1.1) against the characteristic functions supported on a symmetric band or a small cap of the cone, the following conjecture on the restriction of the Fourier transform to the cone can be formulated.

Conjecture 1.1 (Linear adjoint cone restriction conjecture). The inequality (1.1) holds with constants depending on $S, n$ and $p, q$ if and only if $q>\frac{2 n}{n-1}$ and $\frac{n+1}{q} \leq$ $\frac{n-1}{p^{\prime}}$.

Córdoba and Stein proved that (1.1) was true under the condition $p=2$ and $q \geq \frac{2(n+1)}{n-1}$ in an unpublished work. Strichartz [8] then extended the results to more general quadratic surfaces. In 1985, Barcelo 1 proved Conjecture 1.1 when $n=2$. A major breakthrough was made in 2001 by Wolff [14, who showed that Conjecture 1.1 was true when $n=3$. This was based on a new bilinear cone restriction estimate, which also gave the current best result $q>\frac{2(n+3)}{n+1}$ in higher dimensions $n \geq 4$. We should remark that all the recent progress on the linear restriction is achieved from the corresponding bilinear restriction estimates, especially the bilinear $L^{2}$-type estimates, $L^{2} \times L^{2} \rightarrow L^{q}$ for some $q \in[1,2]$; more information about the so-called bilinear method and recent ideas of attacking the restriction conjecture such as the reduction to the local restriction estimates, the wave packet decomposition and the induction-on-scales can be found in [13, [1], 14, [12] and [9].

When we restrict the functions supported on the cone $S$ to be cylindrically symmetric, i.e., functions invariant under spatial rotation, the following theorem is our main result in this paper,

Theorem 1.1. Conjecture 1.1 holds for cylindrically symmetric functions supported on the cone in all dimensions.

For the same class of functions but supported on the paraboloid, the author 4. has verified the corresponding conjecture for the paraboloid in all dimensions. The first proof of Theorem 1.1 is along similar lines as in 4, through dyadically decomposing both the frequency and spatial spaces and then establishing a family of dyadical restriction estimates based on the "Fourier-Bessel" formula defined in Section 3 The second proof is inspired by Nicola's argument on the implication of the cone restriction conjecture from the sphere restriction conjecture in 3 . The key ingredient is the use of the strengthening version of the Hausdorff-Young inequality [7. Chapter 4, Corollary 3.16] and the Hölder inequality in Lorentz spaces [2, Chapter 5, Theorem 5.3.1].

Remark 1.2. As in 4], for the cylindrically symmetric functions with dyadical supports, we expect that more estimates are available. This is indeed the case: from Proposition 3.1 and Corollary 3.4, when $f$ is cylindrically symmetric and supported on a subset of the cone $\{(|\xi|, \xi): 1 \leq|\xi| \leq 2\}$, for $q>\frac{2 n}{n-1}$ and $q \geq p^{\prime}$,

$$
\left\|(f d \sigma)^{\vee}\right\|_{L^{q}\left(\mathbf{R} \times \mathbf{R}^{n}\right)} \leq C_{p, q}\|f\|_{L^{p}(S, d \sigma)} .
$$

We note that $q \geq p^{\prime}$ is an improvement over $q \geq \frac{n+1}{n-1} p^{\prime}$.

Remark 1.3. When $S$ is the whole cone instead of a compact subset of the cone, we see that the necessary conditions are strengthened to

$$
q>\frac{2 n}{n-1}, \quad \frac{n+1}{q}=\frac{n-1}{p^{\prime}} .
$$


In this case, on the one hand, Theorem 1.1 guarantees that the cone restriction conjecture 1.1 is true; on the other hand, unlike the situation in Remark 1.2. there are no more estimates available.

This paper is organized as follows. Section 2 is devoted to establishing the standard notation; in Section 3 we present our first proof of Theorem 1.1 via the dyadic restriction estimates; in Section 4 we present another proof by using a strengthening version of the Hausdorff-Young inequality and the Hölder inequality in Lorentz spaces.

\section{Notation}

We will use the notation $X \lesssim Y, Y \gtrsim X$, or $X=O(Y)$ to denote the estimate $|X| \leq C Y$ for some constant $0<C<\infty$, which may depend on $p, q, n$ and $S$, but not on the functions. If $X \lesssim Y$ and $Y \lesssim X$ we will write $X \sim Y$. If the constant $C$ depends on a special parameter other than the above, we shall denote it explicitly by subscripts. For example, $C_{\varepsilon}$ should be understood as a positive constant not only depending on $p, q, n$ and $S$, but also on $\varepsilon$.

By $\mathcal{S}^{n-1}$ we denote the $(n-1)$-dimensional unit sphere, and by $d \mu$ the canonical surface measure of the sphere. We define a dyadic number to be any number $R \in 2^{\mathbf{Z}}$ of the form $R=2^{j}$ where $j$ is an integer. For each dyadic number $R>0$, we define the dyadic annulus in $\mathbf{R}^{n}, A_{R}:=\left\{x \in \mathbf{R}^{n}: R / 2 \leq|x| \leq R\right\}$. By $\mathcal{L}_{N}$, we denote the class of cylindrically symmetric functions dyadically supported on the cone, i.e., functions invariant under spatial rotation and supported on a set of the form $\{(\tau, \xi): N \leq|\xi| \leq 2 N, \tau=|\xi|\}$ with dyadic $N>0$. We define the time-space norm $L_{t}^{q} L_{x}^{r}$ of $f$ on $\mathbf{R} \times \mathbf{R}^{n}$ by

$$
\|f\|_{L_{t}^{q} L_{x}^{r}\left(\mathbf{R} \times \mathbf{R}^{n}\right)}:=\left(\int_{\mathbf{R}}\left(\int_{\mathbf{R}^{n}}|f(t, x)|^{r} d x\right)^{q / r} d t\right)^{1 / q}
$$

with the usual modifications when $q$ or $r$ is equal to infinity, or when the domain $\mathbf{R} \times \mathbf{R}^{n}$ is replaced by a small region of time-space such as $\mathbf{R} \times A_{R}$. When $q=r$, we abbreviate it by $L_{t, x}^{q}$. We define the spatial Fourier transform of $f$ on $\mathbf{R}^{n}$ by $\hat{f}(\xi)=\int_{\mathbf{R}^{n}} f(x) e^{-i x \cdot \xi} d x$. We use $1_{U}$ to denote the characteristic function of the set $U$, i.e., $1_{U}(x):=1$ if $x \in U$, otherwise 0 . For $1 \leq p \leq \infty$, we denote the conjugate exponent of $p$ by $p^{\prime}$, i.e., $1 / p+1 / p^{\prime}=1$.

\section{First proof of Theorem 1.1}

For any cylindrically symmetric function $f$ on the cone, we set $F(|\xi|):=f(|\xi|, \xi)$. We observe that $(f d \sigma)^{\vee}(t, x)$ is also a cylindrically symmetric function. To begin the proof of Theorem 1.1. we investigate the behavior of $(f d \sigma)^{\vee}$ on $\{|x| \leq 1\}$ via the following proposition.

Proposition 3.1. Suppose $f \in \mathcal{L}_{1}$. Then for any $1 \leq p \leq \infty, q \geq \max \left\{2, p^{\prime}\right\}$ and $R \leq 1$, we have

$$
\left\|(f d \sigma)^{\vee}\right\|_{L_{t, x}^{q}\left(\mathbf{R} \times A_{R}\right)} \lesssim R^{\frac{n}{q}}\|f\|_{L^{p}(S, d \sigma)} .
$$


Proof. If we change to polar coordinates, the left-hand side of (3.1) is

$$
\begin{aligned}
& \left(\int_{A_{R}} \int_{\mathbf{R}}\left|\int_{1 \leq|\xi| \leq 2} f(|\xi|, \xi) e^{i(x \cdot \xi+t|\xi|)} \frac{d \xi}{|\xi|}\right|^{q} d t d x\right)^{1 / q} \\
= & \left(\int_{R / 2}^{R} \int_{\mathbf{R}}\left|\int_{1 \leq|\xi| \leq 2} f(|\xi|, \xi) e^{i\left(r e_{1} \cdot \xi+t|\xi|\right)} \frac{d \xi}{|\xi|}\right|^{q} d t r^{n-1} d r\right)^{1 / q} \\
= & \left(\int_{R / 2}^{R} \int_{\mathbf{R}}\left|\int_{I} F(s) s^{n-2} e^{i t s} \int_{\mathcal{S}^{n-1}} e^{i r s e_{1} \cdot \omega} d \mu(\omega) d s\right|^{q} d t r^{n-1} d r\right)^{1 / q} \\
= & \left(\int_{R / 2}^{R} \int_{\mathbf{R}}\left|\int_{I} F(s) s^{n-2} e^{i t s}(d \mu)^{\vee}\left(r s e_{1} \cdot \omega\right) d s\right|^{q} d t r^{n-1} d r\right)^{1 / q},
\end{aligned}
$$

where $I=[1,2], e_{1}=(1,0, \ldots, 0) \in \mathbf{R}^{n}$ and "." denotes the inner product operation in $\mathbf{R}^{n}$. Then from the Hausdorff-Young inequality when $q>2$ or the Plancherel theorem when $q=2$ and using $\left\|(d \mu)^{\vee}\right\|_{L_{\omega}^{\infty}} \lesssim 1$, the left-hand side of (3.1) is further bounded by

$$
R^{\frac{n-1}{q}}\left(\int_{R / 2}^{R}\|F\|_{L^{q^{\prime}}(I)}^{q} d r\right)^{1 / q} \sim R^{\frac{n}{q}}\|F\|_{L^{q^{\prime}}(I)} .
$$

Then by applying the Hölder inequality to raise $q^{\prime}$ to $p$ since $p \geq q^{\prime}$ and noting $\|F\|_{L^{p}(I)} \sim\|f\|_{L^{p}(S, d \sigma)}$, we see that (3.1) follows.

Before investigating the behavior of $(f d \sigma)^{\vee}$ on $|x| \geq 1$, we exploit the cylindrical symmetry of $f$ in the following proposition. Note that we will encode the error term of the Bessel function into integrals instead of using its asymptotic bound.

Lemma 3.2 (Fourier-Bessel formula). Suppose $f$ is a cylindrically symmetric function supported on the cone. Then

$$
\begin{aligned}
& (f d \sigma)^{\vee}(t, x) \\
& =c_{n} r^{-\frac{n-1}{2}} \int_{I} F(s) s^{\frac{n-3}{2}} e^{i(r s+t s)} d s+c_{n} r^{-\frac{n-1}{2}} \int_{I} F(s) s^{\frac{n-3}{2}} e^{i(-r s+t s)} d s \\
& \quad+c_{n} \int_{I} F(s) s^{n-2} e^{i t s-i r s} \int_{0}^{\infty} e^{-r s y} y^{\frac{n-3}{2}}\left[(y+2 i)^{\frac{n-3}{2}}-(2 i)^{\frac{n-3}{2}}\right] d y d s \\
& \quad+c_{n} \int_{I} F(s) s^{n-2} e^{i t s+i r s} \int_{0}^{\infty} e^{-r s y} y^{\frac{n-3}{2}}\left[(y-2 i)^{\frac{n-3}{2}}-(-2 i)^{\frac{n-3}{2}}\right] d y d s,
\end{aligned}
$$

where I denotes the interval in the radial direction and $r=|x|$.

Proof. We first expand $(f d \sigma)^{\vee}$ in the polar coordinates,

$$
(f d \sigma)^{\vee}(t, x)=\int_{\{|\xi| \in I\}} f(|\xi|, \xi) e^{i\left(r e_{1} \cdot \xi+t|\xi|\right)} \frac{d \xi}{|\xi|}=\int_{I} F(s) e^{i t s} s^{n-2}(d \mu)^{\vee}\left(r s e_{1} \cdot \omega\right) d s .
$$


We recall $(d \mu)^{\vee}(\xi)=c_{n}|\xi|^{\frac{2-n}{2}} J_{\frac{n-2}{2}}(|\xi|)$; see, e.g., [6, page 347]. Moreover from [7. Chapter 3, Lemma 11], we obtain that, for fixed $m \geq 0$,

$$
\begin{aligned}
J_{m}(r) & =c_{m} r^{-1 / 2}\left(e^{i r}-e^{-i r}\right) \\
& +c_{m} r^{m} e^{-i r} \int_{0}^{\infty} e^{-r y} y^{\frac{2 m-1}{2}}\left[(y+2 i)^{\frac{2 m-1}{2}}-(2 i)^{\frac{2 m-1}{2}}\right] d y \\
& +c_{m} r^{m} e^{i r} \int_{0}^{\infty} e^{-r y} y^{\frac{2 m-1}{2}}\left[(y-2 i)^{\frac{2 m-1}{2}}-(-2 i)^{\frac{2 m-1}{2}}\right] d y .
\end{aligned}
$$

Then Lemma 3.2 follows after we combine these two estimates and set $m=\frac{n-2}{2}$.

In view of the previous lemma, we thus define the main term and the error term of $(f d \sigma)^{\vee}$ by

$$
\begin{aligned}
\mathcal{M} f(t, x) & :=c_{n} r^{-\frac{n-1}{2}} \int_{I} F(s) s^{\frac{n-3}{2}} e^{i(r s+t s)} d s+c_{n} r^{-\frac{n-1}{2}} \int_{I} F(s) s^{\frac{n-3}{2}} e^{i(-r s+t s)} d s, \\
\mathcal{E} f(t, x) & :=c_{n} \int_{I} F(s) s^{n-2} e^{i t s+i r s} \int_{0}^{\infty} e^{-r s y} y^{\frac{n-3}{2}}\left[(y+2 i)^{\frac{n-3}{2}}-(2 i)^{\frac{n-3}{2}}\right] d y d s \\
& -c_{n} \int_{I} F(s) s^{n-2} e^{i t s-i r s} \int_{0}^{\infty} e^{-r s y} y^{\frac{n-3}{2}}\left[(y-2 i)^{\frac{n-3}{2}}-(-2 i)^{\frac{n-3}{2}}\right] d y d s .
\end{aligned}
$$

Heuristically, one should think of $\mathcal{E} f$ as $r^{-(n+1) / 2} \int_{I} F(s) s^{\frac{n-5}{2}} e^{i t s} d s$, which is given by estimating the error term of the Bessel function $J_{m}(r)$ by $r^{-3 / 2}$. The following proposition shows that the error term estimate is acceptable compared to the main term estimate.

Proposition 3.3. Suppose $f \in \mathcal{L}_{1}$. Then for all $1 \leq p \leq \infty, q \geq \max \left\{2, p^{\prime}\right\}$, a dyadic number $R \geq 2$ and $f \in L^{p}(S, d \sigma)$, we have the main term estimate,

$$
\|\mathcal{M} f\|_{L_{t, x}^{q}\left(\mathbf{R} \times A_{R}\right)} \lesssim R^{-\frac{n-1}{2}\left[1-\frac{2 n}{q(n-1)}\right]}\|f\|_{L^{p}(S, d \sigma)},
$$

and the error term estimate

$$
\|\mathcal{E} f\|_{L_{t, x}^{q}\left(\mathbf{R} \times A_{R}\right)} \lesssim R^{-\frac{n+1}{2}+\frac{n}{q}}\|f\|_{L^{p}(S, d \sigma)} .
$$

Proof. To prove the main term estimate (3.2), we first observe that it is sufficient to obtain the same estimate with the first term in the expression of $\mathcal{M} f$. Then by changing to polar coordinates and the Hausdorff-Young inequality in $t$ when $q>2$ or the Plancherel theorem in $t$ when $q=2$, we obtain

$$
\begin{aligned}
\|\mathcal{M} f\|_{L_{t, x}^{q}\left(\mathbf{R} \times A_{R}\right)} & \sim\left(\int_{R / 2}^{R} \int_{\mathbf{R}}\left|r^{-\frac{n-1}{2}} \int_{I} F(s) s^{\frac{n-3}{2}} e^{i(r s+t s)} d s\right|^{q} d t r^{n-1} d r\right)^{1 / q} \\
& =R^{-\frac{n-1}{2}+\frac{n-1}{q}}\left(\int_{R / 2}^{R} \int_{\mathbf{R}}\left|\int_{I} F(s) s^{\frac{n-3}{2}} e^{i r s} e^{i t s} d s\right|^{q} d t d r\right)^{1 / q} \\
& \lesssim R^{-\frac{n-1}{2}+\frac{n-1}{q}}\left(\int_{R / 2}^{R}\|F\|_{L_{s}^{q^{\prime}}(I)}^{q} d r\right)^{1 / q} \lesssim R^{-\frac{n-1}{2}+\frac{n}{q}}\|f\|_{L^{p}(S, d \sigma)} .
\end{aligned}
$$

Hence (3.2) follows.

To prove the error term estimate (3.3), for $r \geq 1$, we set

$$
E(r)=\int_{0}^{\infty} e^{-r y} y^{\frac{n-3}{2}}\left[(y \pm 2 i)^{\frac{n-3}{2}}-( \pm 2 i)^{\frac{n-3}{2}}\right] d y .
$$


By an argument similar to that used in proving [7, Chapter 3, Lemma 3.11] (details can also be found in [4, Proposition 3.3]), we have

$$
|E(r)| \lesssim r^{-\frac{n+1}{2}}, \text { for } r \geq 1 .
$$

By changing to polar coordinates, the left-hand side of (3.3) is comparable to

$$
\left(\int_{R / 2}^{R} \int_{\mathbf{R}}\left|\int_{I} F(s) s^{n-2} e^{i t s \pm i r s} E(r s) d s\right|^{q} d t r^{n-1} d r\right)^{1 / q} .
$$

Then by the Hausdorff-Young inequality in $t$ when $q>2$ or the Plancherel theorem in $t$ when $q=2$ and $s \sim 1$, it is further bounded by

$$
\left(\left.\left.\int_{R / 2}^{R}\left|\int_{I}\right| F(s) s^{n-2} E(r s)\right|^{q^{\prime}} d s\right|^{q / q^{\prime}} r^{n-1} d r\right)^{1 / q} .
$$

By using (3.4) and Hölder since $q \geq p^{\prime}$, it is bounded by $R^{-\frac{n+1}{2}+\frac{n}{q}}\|F\|_{L^{p}(I)}$. Then (3.3) follows because $\|F\|_{L^{p}(I)} \sim\|f\|_{L^{p}(S, d \sigma)}$.

From the triangle inequality, we have

Corollary 3.4 (Dyadic restriction estimate). Suppose $f \in \mathcal{L}_{1}$. Then for all $1 \leq$ $p \leq \infty, q \geq \max \left\{2, p^{\prime}\right\}$, a dyadic number $R \geq 2$ and $f \in L^{p}(S, d \sigma)$, we have

$$
\left\|(f d \sigma)^{\vee}\right\|_{L_{t, x}^{q}\left(\mathbf{R} \times A_{R}\right)} \lesssim R^{-\frac{n-1}{2}\left[1-\frac{2 n}{q(n-1)}\right]}\|f\|_{L^{p}(S, d \sigma)} .
$$

Having done all the preparations, we now prove Theorem 1.1 via the dyadic restriction estimate above.

The first proof of Theorem 1.1. We only need to show the "sufficient" part of the claim. We first observe that it suffices to prove (1.1) under the boundary condition $q>\frac{2 n}{n-1}$ and $\frac{n+1}{q}=\frac{n-1}{p^{\prime}}$ since other estimates are easily obtained by a standard argument of using the Hölder inequality. From Corollary 3.4 and Proposition 3.1 . we obtain that, for $q>\frac{2 n}{n-1}, \frac{n+1}{q}=\frac{n-1}{p^{\prime}}$, and $f \in \mathcal{L}_{1}$,

$$
\left\|(f d \sigma)^{\vee}\right\|_{L_{t, x}^{q}\left(\mathbf{R} \times A_{R}\right)} \lesssim R^{\alpha(R)}\|f\|_{L^{p}(S, d \sigma)},
$$

where

$$
\alpha(R)= \begin{cases}-\frac{n-1}{2}\left[1-\frac{2 n}{q(n-1)}\right], & \text { for } R \geq 2, \\ \frac{n-1}{q}, & \text { for } R \leq 1 .\end{cases}
$$

By scaling, when $f \in \mathcal{L}_{M}$ with $M \in 2^{\mathbf{Z}}$, under the condition $\frac{n+1}{q}=\frac{n-1}{p^{\prime}}$,

$$
\left\|(f d \sigma)^{\vee}\right\|_{L_{t, x}^{q}\left(\mathbf{R} \times A_{R}\right)} \lesssim(R M)^{\alpha(R M)}\|f\|_{L^{p}(S, d \sigma)} .
$$

Then for general $f$, we decompose it as follows:

$$
f=\sum_{M: d y a d i c} f 1_{\{(\tau, \xi): \tau=|\xi|, M \leq|\xi| \leq 2 M\}}=\sum_{M} f_{M},
$$


where $f_{M}:=f 1_{\{(\tau, \xi): \tau=|\xi|, M \leq|\xi| \leq 2 M\}}$. Hence

$$
\begin{aligned}
& \left\|(f d \sigma)^{\vee}\right\|_{L_{t, x}^{q}\left(\mathbf{R} \times \mathbf{R}^{n-1}\right)}=\left(\sum_{R}\left\|(f d \sigma)^{\vee}\right\|_{L_{t, x}^{q}\left(\mathbf{R} \times A_{R}\right)}^{q}\right)^{1 / q} \\
& =\left(\sum_{R}\left\|\sum_{M}\left(f_{M} d \sigma\right)^{\vee}\right\|_{L_{t, x}^{q}\left(\mathbf{R} \times A_{R}\right)}^{q}\right)^{1 / q} \lesssim\left(\sum_{R}\left(\sum_{M}\left\|\left(f_{M} d \sigma\right)^{\vee}\right\|_{L_{t, x}^{q}\left(\mathbf{R} \times A_{R}\right)}\right)^{q}\right)^{1 / q} \\
& \lesssim\left(\sum_{R}\left(\sum_{M}(R M)^{\alpha(R M)}\left\|f_{M}\right\|_{L^{p}(S, d \sigma)}\right)^{q}\right)^{1 / q} \lesssim\left(\sum_{M}\left\|f_{M}\right\|_{L^{p}(S, d \sigma)}^{p}\right)^{1 / p} \sim\|f\|_{L^{p}(S, d \sigma)},
\end{aligned}
$$

where $R>0$ and $M>0$ are dyadic numbers; for the last line, we have used the Schur test since $q>\frac{2 n}{n-1}>p \geq 1$ and

$$
\sup _{R>0} \sum_{M}(R M)^{\alpha(R M)}<\infty \text { and } \sup _{M>0} \sum_{R}(R M)^{\alpha(R M)}<\infty .
$$

Hence Theorem 1.1 follows.

\section{Second proof of Theorem 1.1}

To begin the second proof, we introduce the following strengthening version of the Hausdorff-Young inequality [7, Chapter 4, Corollary 3.16].

Lemma 4.1. If $f \in L^{p}\left(\mathbf{R}^{n}\right), 1<p \leq 2$, then $\hat{f}$ belongs to $L^{p, p^{\prime}}$ and

$$
\|\hat{f}\|_{L^{p^{\prime}, p}} \lesssim\|f\|_{L^{p}}
$$

or in its dual form, for any $f \in L^{p, p^{\prime}}$,

$$
\|\hat{f}\|_{L^{p^{\prime}}} \lesssim\|f\|_{L^{p, p^{\prime}}}
$$

where $L^{p, q}$ denotes the Lorentz space for $0<p<\infty, 0<q \leq \infty$, which is defined via the equivalence that $f \in L^{p, q}$ if and only if the norm $\|f\|_{L^{p, q}}:=$ $\left(\frac{q}{p} \int_{0}^{\infty}\left(\lambda\left|\left\{x \in \mathbf{R}^{n}:|f(x)|>\lambda\right\}\right|^{1 / p}\right)^{q} \frac{d \lambda}{\lambda}\right)^{1 / q}$ is finite with the usual modification weak- $L^{p}$ when $q=\infty$.

We also introduce the following Hölder inequality in the Lorentz spaces [2, Chapter 5, Theorem 5.3.1].

Lemma 4.2. If $0<p_{1}, p_{2}, p<\infty$ and $0<q_{1}, q_{2}, q \leq \infty$ obey $\frac{1}{p}=\frac{1}{p_{1}}+\frac{1}{p_{2}}$ and $\frac{1}{q}=\frac{1}{q_{1}}+\frac{1}{q_{2}}$, then

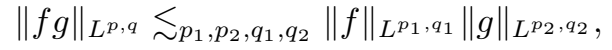

whenever the right-hand side norms are finite.

Next we will present the second proof of Theorem 1.1, which is inspired by Nicola's short proof in [3] that the restriction conjecture for the sphere in $\mathbf{R}^{n}$ implies that for the cone in $\mathbf{R} \times \mathbf{R}^{n}$.

The second proof of Theorem 1.1. As in the first proof, it is sufficient to consider $q>\frac{2 n}{n-1}$ and $\frac{n+1}{q}=\frac{n-1}{p^{\prime}}$. By changing to polar coordinates,

$$
(f d \sigma)^{\vee}(t, x)=c_{n} r^{-\frac{n-2}{2}} \int_{0}^{\infty} e^{i t s} F(s) s^{\frac{n-2}{2}} J_{\frac{n-2}{2}}(s r) d s .
$$


Then by using Lemma 4.1 and exchanging the norms, we have

$$
\begin{aligned}
\left\|(f d \sigma)^{\vee}\right\|_{L_{t, x}^{q}} & \lesssim\|\| r^{-\frac{n-2}{2}+\frac{n-1}{q}} F(s) s^{\frac{n}{2}-1} J_{\frac{n}{2}-1}(s r)\left\|_{L_{s}^{q^{\prime}, q}(0, \infty)}\right\|_{L_{r}^{q}(0, \infty)} \\
& \lesssim\left\|F(s) s^{\frac{n}{2}-1}\right\| r^{-\frac{n-2}{2}+\frac{n-1}{q}} J_{\frac{n}{2}-1}(r s)\left\|_{L_{r}^{q}(0, \infty)}\right\|_{L_{s}^{q^{\prime}, q}(0, \infty)} .
\end{aligned}
$$

We observe that for each $s>0$, the integrand is bounded by

$$
\left\|r^{-\frac{n-2}{2}+\frac{n-1}{q}} J_{\frac{n}{2}-1}(r s)\right\|_{L_{r}^{q}(1 / s, \infty)}+\left\|r^{-\frac{n-2}{2}+\frac{n-1}{q}} J_{\frac{n}{2}-1}(r s)\right\|_{L_{r}^{q}(0,1 / s)} .
$$

On the one hand, from the definition of the Bessel function

$$
J_{\frac{n}{2}-1}(r)=\frac{(r / 2)^{\frac{n-2}{2}}}{\Gamma((n-1) / 2) \Gamma(1 / 2)} \int_{-1}^{1} e^{i r s}\left(1-s^{2}\right)^{\frac{n-3}{2}} d s,
$$

we obtain

$$
J_{\frac{n}{2}-1}(r) \lesssim r^{\frac{n-2}{2}} \text { for } n \geq 2 \text { and } r \leq 1 .
$$

On the other hand, from the complete expansion of $J_{m}$ when $m=\frac{n}{2}-1$ and the bound on $E(r)$ in the proof of Proposition 3.3 , we have

$$
\left|J_{\frac{n}{2}-1}(r)\right| \lesssim r^{-1 / 2}+c_{n} r^{\frac{n}{2}-1} r^{-\frac{n+1}{2}} \lesssim r^{-1 / 2}, \text { for } n \geq 2 \text { and } r \geq 1 .
$$

Hence combining these two estimates on $J_{\frac{n}{2}-1}$, we obtain

$$
\text { (4.1) } \lesssim s^{\frac{n}{2}-\frac{n}{q}-1} \text { if } q>\frac{2 n}{n-1} \text {. }
$$

Then by the fact that $q>p$ and Lemma 4.2 .

$$
\begin{aligned}
\left\|(f d \sigma)^{\vee}\right\|_{L_{t, x}^{q}} & \lesssim\left\|F(s) s^{\frac{n-2}{p}} s^{-\frac{n-2}{p}+n-\frac{n}{q}-2}\right\|_{L_{s}^{q^{\prime}, q}} \\
& \lesssim\left\|F(s) s^{\frac{n-2}{p}} s^{-\frac{n-2}{p}+n-\frac{n}{q}-2}\right\|_{L_{s}^{q^{\prime}, p}} \\
& \lesssim\left\|F(s) s^{\frac{n-2}{p}}\right\|_{L^{p, p}\left\|s^{-\frac{n-2}{p}+n-\frac{n}{q}-2}\right\|_{L^{\frac{1}{1 / q^{\prime}-1 / p}, \infty}}} .
\end{aligned}
$$

Note the condition $\frac{n+1}{q}=\frac{n-1}{p^{\prime}}$ implies that $-\frac{n-2}{p}+n-\frac{n}{q}-2=-\left(\frac{1}{q^{\prime}}-\frac{1}{p}\right)$. Hence

$$
\left\|s^{-\frac{n-2}{p}+n-\frac{n}{q}-2}\right\|_{L^{\frac{1}{1 / q^{\prime}-1 / p}}, \infty}<\infty .
$$

Therefore, by the fact that $\left\|F(s) s^{\frac{n-2}{p}}\right\|_{L^{p, p}}=\|f\|_{L^{p}(S, d \sigma)}$, we see that Theorem 1.1 follows.

\section{ACKNOWLEDGMEnTs}

The author is very grateful to his advisor, Terence Tao, for helpful discussions on this problem and for his support during the preparation of this paper. The author would also like to thank the referee for valuable suggestions and comments.

\section{REFERENCES}

[1] B. Barcelo Taberner, On the restriction of the Fourier transform to a conical surface, Trans. Amer. Math. Soc. 292 (1985), no. 1, 321-333. MR805965 (86k:42023)

[2] Jöran Bergh and Jörgen Löfström, Interpolation spaces. An introduction, Springer-Verlag, Berlin, 1976, Grundlehren der Mathematischen Wissenschaften, No. 223. MR0482275 $(58: 2349)$

[3] F. Nicola, Slicing surfaces and Fourier restriction conjecture, arXiv:0804.3696, Proceedings of the Edinburgh Mathematical Society, to appear.

[4] S. Shao, Sharp linear and bilinear restriction estimates for paraboloids in the cylindrically symmetric case, arXiv:0706.3759, Revista Matemática Iberoamericana, to appear. 
[5] E. M. Stein, Some problems in harmonic analysis, Harmonic analysis in Euclidean spaces (Proc. Sympos. Pure Math., Williams Coll., Williamstown, Mass., 1978), Part 1, Proc. Sympos. Pure Math., XXXV, Part, Amer. Math. Soc., Providence, RI, 1979, pp. 3-20. MR.545235 (80m:42027)

[6] - Harmonic analysis: Real-variable methods, orthogonality, and oscillatory integrals, Princeton Mathematical Series, vol. 43, Princeton University Press, Princeton, NJ, 1993, With the assistance of Timothy S. Murphy, Monographs in Harmonic Analysis, III. MR.1232192 (95c:42002)

[7] E. M. Stein and G. Weiss, Introduction to Fourier analysis on Euclidean spaces, Princeton University Press, Princeton, NJ, 1971, Princeton Mathematical Series, No. 32. MR 0304972 (46:4102)

[8] R. S. Strichartz, Restrictions of Fourier transforms to quadratic surfaces and decay of solutions of wave equations, Duke Math. J. 44 (1977), no. 3, 705-714. MR0512086 (58:23577)

[9] T. Tao, Recent progress on the restriction conjecture, arXiv:math/0311181.

[10] _ The Bochner-Riesz conjecture implies the restriction conjecture, Duke Math. J. 96 (1999), no. 2, 363-375. MR1666558 (2000a:42023)

[11] Endpoint bilinear restriction theorems for the cone, and some sharp null form estimates, Math. Z. 238 (2001), no. 2, 215-268. MR1865417(2003a:42010)

[12] _ A sharp bilinear restrictions estimate for paraboloids, Geom. Funct. Anal. 13 (2003), no. 6, 1359-1384. MR2033842 (2004m:47111)

[13] T. Tao, A. Vargas, and L. Vega, A bilinear approach to the restriction and Kakeya conjectures, J. Amer. Math. Soc. 11 (1998), no. 4, 967-1000. MR1625056 (99f:42026)

[14] T. Wolff, A sharp bilinear cone restriction estimate, Ann. of Math. (2) 153 (2001), no. 3, 661-698. MR.1836285 (2002j:42019)

Department of Mathematics, University of California, los Angeles, California 90095- 1555

Current address: School of Mathematics, Institute for Advanced Study, Einstein Drive, Princeton, New Jersey 08540

E-mail address: slshao@math.ucla.edu 